ISAHP 2001, Berne, Switzerland, August 2-4, 2001

\title{
A UNIT INTERPRETATION OF MULTI-CRITERIA RATIOS
}

\author{
William C. Wedley and Eng Ung Choo ${ }^{1}$ \\ Faculty of Business Administration \\ Simon Fraser University \\ Burnaby, B. C. Canada, V5A 1S6 \\ wedley@sfu.ca, choo@sfu.ca
}

Keywords: AHP ratios, natural zero, unit of measure

Summary: When AHP ratios are normalized to sum to unity, the unit of measure becomes obscure. This paper investigates this obscurity and whether ratio measurement is possible when there is no prior knowledge of the measurement unit. First, we look at ratio scales of tangible attributes of objects with well-known measures. Then ratio scales of unknown intangible attributes of objects are analyzed. We discover that natural zero and the unit of measure are not necessarily used explicitly in deriving ratio scale measures. Nevertheless, the derived scale does have a derived unit of measurement. We conclude that although composite multi-criteria answers are possible in ratio form, it is important to know that a unit of measure exists if ambiguities are to be avoided.

\section{Introduction}

The Analytic Hierarchy Process has been criticized and misunderstood by many academician and practitioners. Barzilai (1998) has been one of the strongest critics of AHP, claiming that ratio measurements are impossible and that the process is irreparably flawed. Saaty (1999) on the other hand, claims that a relative ratio scale does not need a unit of measurement. Others claim that AHP produces dimensionless units once the original measures are converted to priorities. This paper looks at these two issues and claims that AHP priorities do have a unit of measure and that the process is not flawed. The paper approaches the issues by first looking at ratio scale measures of well-known tangible attributes and then ratio scale measures of unknown intangible attributes. With this knowledge as background, we then look at whether specific knowledge of natural zero is necessary to generate ratio scales and composite, multi-criteria answers that are in ratio form.

\section{The Nature of Ratio Scales}

\subsection{Ratio Scales of Measurable Elements}

A numerical measuring scale for an attribute of a set of objects is said to be in ratio scale whenever the measures are not affected by multiplication of any positive constant. Let $\mathrm{a}_{\mathrm{i} j \mathrm{j}}$ be the paired comparison of an attribute of objects $y_{i}$ to $y_{j}$ from the ratio scale $y=b x$ where the unit of measure, $x$, is known or unknown. Our notation, $\mathrm{a}_{\mathrm{i} j \mathrm{j}}$, is slightly different from the usual $\mathrm{a}(\mathrm{i}, \mathrm{j})$ notation used when referring to AHP comparisons, because we want to emphasize that one of the pairs (j) is used as the referent or unit of comparison. When making AHP comparisons, the questioning procedure requires two questions -- first, which attribute value possessed by an object is more desirable or important and second, by how many times. The less dominant object of the comparison determined by the first question becomes the referent ( $\mathrm{j}$ )

\footnotetext{
${ }^{1}$ Acknowledgement: The authors thank the Natural Sciences and Engineering Research Council of Canada
} for financial support related to this project.

Proceedings $-6^{\text {th }}$ ISAHP 2001 Berne, Switzerland 
and the second question determines the intensity. Once $\mathrm{a}_{\mathrm{i} j \mathrm{j}}$ is determined, $\mathrm{a}_{\mathrm{j} \mathrm{i}}$ is found by the reciprocal relationship $\mathrm{a}_{\mathrm{j} / \mathrm{i}}=1 / \mathrm{a}_{\mathrm{i} / \mathrm{j}}$.

The big concern with these two comparison questions is whether the decision-maker is able to answer them meaningfully. This capability is a huge presumption, because it eliminates the need for natural zero and explicit knowledge of the unit of measure.

When the unit of measure is known, weight in kilograms, for example, the coefficient $b$ determines the number of units of the measure and $a_{i j j}=y_{i} / y_{j}=b_{i} x / b_{j} x$. Therefore, the ratio of the weight of an object of 20 kilograms to an object of 10 kilograms is $20 / 10=20(1) / 10(1)=2 / 1$. The denominator item, the 10 kilogram weight, is the unit of measure.

In ratio scales, multiplication by any positive constant is a permissible transformation to a different scale representing a new unit of measure. For example, multiplying weights measured in kilograms by $1 / 2$ converts the weights in the kilogram scale to the weights on a half-kilogram scale. Similarly, multiplying weight on the kilogram scale by 2.2 or $1 / .454$ converts the weight to a scale measured in pounds. Although the units of measure have changed, the relative ratios have not -- $(20 / .454) /(10 / .454)$ is still $2 / 1$. In effect, the choice of unit of measure in ratio scales is arbitrary (Barzilai, 1998). Many different units can be used.

When we have a tangible attribute of several objects measured objectively by a ratio scale (say 10, 20, 50 kilograms), the attribute of any one of these objects could be used as the unit of measure. For example, columns of Table 1 express the weights of 10,20 and 50-kilogram objects in different scales. Each column represents measurement on a different scale, although the relative ratio of the weights of any two objects on any of those scales remains unchanged.

Table 1 -- Three objects expressed in different weight units

\begin{tabular}{|c|c|c|c|c|c|c|}
\hline & Case 1 & Case 2 & Case 3 & Case 4 & Case 5 & Case 6 \\
\hline Unit $=$ & $1 \mathrm{Kg}$. & $1 \mathrm{Lb}$. & $10 \mathrm{Kg}$. & $20 \mathrm{Kg}$. & $50 \mathrm{Kg}$. & Total Kg. \\
\hline $\mathbf{A}$ & $10 / 1=10$ & $10 * 2.2=22$ & $10 / 10=1$ & $10 / 20=.5$ & $10 / 50=.2$ & $10 / 80=.125$ \\
\hline B & $20 / 1=20$ & $20 * 2.2=44$ & $20 / 10=2$ & $20 / 20=1$ & $20 / 50=.4$ & $20 / 80=.250$ \\
\hline C & $50 / 1=50$ & $50 * 2.2=110$ & $50 / 10=5$ & $50 / 20=2.5$ & $50 / 50=1$ & $50 / 80=.625$ \\
\hline Referent $=$ & $1 \mathrm{KG}$ & $.454 \mathrm{KG}$ & A & B & $\mathrm{C}$ & $80 \mathrm{Kgs}$. \\
\hline
\end{tabular}

In effect, the paired comparison of attribute measure $y_{i}=b x$ to that of a common referent object $y_{R}$ converts the unit of measure to the attribute measure of the referent object, $y_{i R}=b x / y_{R}$. For any such conversion, the ratio between the attribute measures of any two objects $\mathrm{j}$ and $\mathrm{k}$ remains unchanged. For example, the ratio of the weights of $\mathrm{B}$ to $\mathrm{C}$ remains .4 no matter which of the previous scales is used.

In a paired comparison matrix $\mathrm{A}$, each column represents a separate transformation of the original measurement scale to units of $y_{i R}$, the attribute measure of the column referent object. In Table 1, the boldfaced entries with diagonals equal to unity represent the paired comparisons of an AHP matrix. Assuming accuracy and consistency in the paired comparison judgments, the ratio between any attribute measure in the columns remains unchanged, even when, as exists here, the units of measure of each column are different.

Within any such column of the consistent paired comparison matrix, a further permissible transformation is to divide each column attribute measure by the sum of all the column attribute measures. Such a transformation process (multiplication by a constant) is a type of normalization that converts all columns to 
common scales that sum to unity. Although all columns would now be in common units, the original units for each column are lost if the normalization factor cannot be recovered (just as we cannot convert any of the scales in Table 1 back to kilograms if we do not know $y_{R}$, the unit of measure of the referent for the column). We also note that if the size of the matrix is changed by adding or deleting objects, a new normalization that re-sums all column attribute measures to unity will yield new common scales for the columns, but not in the same units as the original normalized matrix.

As pointed out by Saaty and Vargas (1993), the normalized principal eigenvector of the consistent pairwise comparison matrix is the same as the common column scales normalized to sum to one. Thus, the eigenvector or the common column scales can be looked upon as a ratio scale measured in units representing the sum of the compared attribute measures. Different sums of attribute measures, therefore, like different referent objects, represent different units. In other words, different normalizations represent different units of measure.

Notice that in the above discussion of ratio scales from pairwise comparisons, no mention was made to the existence of natural zero. So far, that issue has been ignored. A requirement of ratio scales, however, is the existence of a natural zero that represents the absence of measurement sensation. In other words, a ratio scale can be represented by $\mathrm{y}=\mathrm{a}+\mathrm{bx}$ where $\mathrm{a}=$ natural zero. In our example, it would be an object weighing 0.000000 kilograms.

A pertinent question is whether natural zero must be known before a ratio scale can be defined. Barzilai (1998), for example, has correctly noted that ratio scales (or unit scales as he calls them) are scales where natural zero is fixed and the only free parameter is the unit. Barzilai contends, however, that the implied assumption of a natural zero is insufficient and that the unit difference away from natural zero must be known before meaningful comparisons can be made. It is contended herein that while it is necessary to either measure or sense the unit difference away from natural zero, in deriving ratio scales, it is immaterial what that unit is.

Consider an element whose measurement is greater than natural zero by $\varepsilon$, an imperceptible small amount. If $\varepsilon$ is used as the unit of measure as compared to the attribute measures of other objects higher on the measurement scale and if $\varepsilon$ cannot be sensed and perceived, then the comparison yi/ $\varepsilon$ would be infinity. In effect, $\varepsilon$ being imperceptible and more than one order of magnitude away from the other objects is similar to natural zero. Like $\varepsilon$, natural zero does not play a meaningful role in paired comparisons.

Assume that one wants to determine the relative weights of three objects but is not told their units in kilograms or pounds. Not knowing the unit of measurement, one could use the first object as the unit and come up with the ratios $y_{2} / y_{1}=2, y_{3} / y_{1}=5$. Since $y_{1} / y_{1}=1$, the relative weights in terms of $y_{1}$ units are 1 , 2 and 5 for $y_{1}$ to $y_{3}$ respectively. Alternatively, normalizing so that the unit is the sum of the elements, the ratios are $.125, .250$ and .625 . We note that these are the same ratios as were derived from objects weighing 10, 20 and 50 kilograms. One should not conclude that the original units were measured in kilograms, because the same ratios could have come from 2, 4 and 10 pounds or a plum, an orange and a watermelon.

\subsection{Ratio Scales of Preferences from Elements without Measures}

We have presumed that the decision-maker is capable of deriving ratio scales of attribute measures such as weights of objects even when we do not know the unit of the scale used to measure the attribute. In order to make meaningful comparisons, we must be able to sense the attribute of the objects being compared, which implies that the attribute of those objects are not at natural zero. It also implies that we need to be able to express the strength of the attributes of objects in terms of the number of times one exceeds the other (assuming the lower attribute measure becomes the unit of comparison).

For attributes similar to weight (jogging distance, time, area, space, etc.) for which well-known objective measurement scales are available, it is relatively easy to perceive how ratio scales could be derived. For other attributes of objects such as prestige, comfort, safety and quality, measurement information may be 
unavailable (prestige, comfort) or the measurement information may be partial or in non-ratio form (safety, quality). Under such situations, is it possible to measure preferences in ratio form?

We will approach this problem by looking at a known attribute, say jogging distance. We will evaluate a person's preference for distance rather than the actual physical measurement of it. Consider a person who regularly exercises by jogging an ideal distance of 4 kilometres per day. The person travels to a foreign country and finds a system of jogging paths that are marked on a map. The map is in a language the person cannot understand. The routes are discernible, but not the distances. What would this person's preference measures look like?

Presumably, the person could try a different path each day and specify (1) which path is preferred (an ordinal measure) and (2) by how many times (a ratio measure). If done correctly, paths on either side of 4 kilometres would be less preferred. If perchance one route is trivially short, say 200 metres, the person would be comparing other relevant routes to the 200 metre one that provides zero preference. In this case, the 200-metre route would be like natural zero. And like natural zero, other routes compared to it would be infinitely preferred. Note that "zero distance" on the physical measurement scale of distance may not be the natural zero of the preference measurement scale.

But what happens when one of the jogging routes is much greater than 4 kilometres. Eventually, that distance would be so long that the person would be unable to complete it. At some such distance, the too long distance would have zero preference and also be equivalent to natural zero. This implies that although there is exactly one natural zero on a preference measurement scale, it may be the preference measure for two or more distances.

\subsection{Ratio Scales of Preferences Across Preference Scales}

In multi-criteria decision problems, scales of measure from several separate criteria are aggregated into a composite numerical score. The most common aggregation is via linear weighted addition. Fundamental to the process is whether the aggregation from ratio scales, as in the AHP, can also be in ratio form. In other words, are the composite (added) attribute measures from the same underlying scale and therefore in commensurate terms?

Essential to the multi-criteria problem is the fact that each criterion represents a different dimension and different unit of measure. Even though the aggregation of these different units is very different from the transformation of a ratio scale by adding some constant, it is not clear that these different units can be aggregated into a composite unit that is still in ratio scale. In short, how can aggregation be accomplished when addition is not a permissible transformation of a ratio scale? The answer lies in the concept of scaling and commensurability.

To illustrate the multi-criteria situation, we will utilize a suitcase purchase problem where there are only two criteria (maximum carrying weight and style) for evaluating three alternatives (suitcases A, B and C). The maximum carrying weights are determined objectively from the product specifications. For the purposes of this problem, we will assume they are 10,20 and 50 kilograms respectively, the same as in Table 1. The style of each suitcase is not measured objectively. Rather, it is a subjective criterion, being based upon the personal preference of the decision-maker.

Even though weight is measured objectively, we do not necessarily have linear utility for weight. For example, the 50 kilogram capacity for suitcase $\mathrm{C}$ is preferred more than the 20 kilogram capacity of suitcase $\mathrm{B}$, but not necessarily $50 / 20=2.5$ times. After the suitcase carrying capacity reaches 30 kilograms, we may consider the incremental amounts of capacity to have diminishing utility, because we cannot visualize ourselves filling or lifting a suitcase that has more than 20 kilograms of weight. Accordingly, we may conclude that the capacity of suitcase $\mathrm{C}$ is twice as preferred as that of suitcase B. Conversely, a suitcase with only 10 kilograms of carrying capacity would be relatively impractical. We may say that the capacity of suitcase B is 4 times as desirable as that of suitcase A (or, being consistent, capacity of suitcase $\mathrm{C}$ is 8 times as preferred). 
For the weight criterion, it is clear that even though there is an objective measure with which we are familiar, we may not have linear preference for that measure. It depends upon the circumstances and our preference. For style, the same principle applies, although there is no well-known objective measure like weight in the background. So long as we are able to express our ordinal preference (which one is preferred) and then the multiplicative intensity of that preference over the other, we can make ratio comparisons that can be captured in a ratio scale. Note that when we are using the fundamental scale to make comparisons with AHP, the compared items are supposed to be within one order of magnitude. Hence, we would never be making comparisons with attribute measures that are at or close to natural zero, because such comparisons would have the more desirable attribute measure infinitely more preferred.

A plausible set of paired comparisons for the alternatives are presented in Table 2. We should note that although these paired comparisons were taken from an underlying scale within one order of magnitude (the fundamental 1-9 scale), the resulting ratio priorities are unlikely to be in overall, commensurate units. This is because, in overall terms, the criteria have different importance to our choice. Only in the very unusual case where all criteria have equal importance would priorities under each criterion be in commensurate units.

Table 2 -- Paired Comparisons and Priorities for Weight Capacity and Style

\begin{tabular}{|c|c|c|c|}
\hline & \multicolumn{3}{|c|}{ Weight Capacity } \\
\hline & $\mathbf{A}$ & B & $\mathrm{C}$ \\
\hline $\mathbf{A}$ & 1 & $1 / 4$ & $1 / 8$ \\
\hline B & 4 & 1 & $1 / 2$ \\
\hline C & 8 & 2 & 1 \\
\hline
\end{tabular}

\begin{tabular}{|c|}
\hline Priorties \\
\hline 0.077 \\
0.308 \\
0.615
\end{tabular}

\begin{tabular}{l|c|c|c|}
\multicolumn{4}{c}{ Style } \\
\multicolumn{1}{c|}{ A } & \multicolumn{1}{c}{ B } & \multicolumn{1}{c|}{ C } \\
\cline { 2 - 4 } A & 1 & 0.5 & 1 \\
\cline { 2 - 4 } B & 2 & 1 & 2 \\
\cline { 2 - 4 } C & 1 & 0.5 & 1 \\
\cline { 2 - 4 }
\end{tabular}

Priorties

0.250

0.500

0.250

In Table 2, we have purposefully kept the comparisons completely consistent so as to emphasize each column as measurement in its own unit. For any column, we can normalize by the sum of its column to transform the attribute measures into priorities that sum to unity. Since this column divisor determines the new unit, it means that the unit of local priorities is the sum of the relative importance of the criterion possessed by the alternatives. Therefore, .077 measures the importance of suitcase A in units of the sum of the local priorities of the weight capacity of the three suitcases and .250 measures the importance of suitcase $\mathrm{A}$ in units of the sum of the local priorities of the style of the three suitcases.

But are units of the summed local priorities of the weight capacities commensurable with the units of the summed local priorities of the style of the three suitcases so that they can be added to get a composite priority? Seldom not! Most often, the criteria differ in their relative importance and therefore in their commensurability. In Table 3, we have specified Style to be 1.5 times (.6/.4) more important than Weight Capacity. When we multiply the local priorities of the alternatives by these criteria weights, we are undertaking another proportional transformation whereby the local priorities of the alternatives are.

Table 3 -- Local, Global and Composite Priorities.

\section{Criteria Priorities}

\begin{tabular}{l|l}
0.4 & 0.6
\end{tabular}

Local Weights

Weight

Capacity

\begin{tabular}{l|c|c|}
\multicolumn{1}{c}{ Capacity } & \multicolumn{1}{c}{ Style } \\
A & 0.077 & 0.250 \\
B & 0.308 & 0.500 \\
C & 0.615 & 0.250 \\
\cline { 2 - 3 }
\end{tabular}

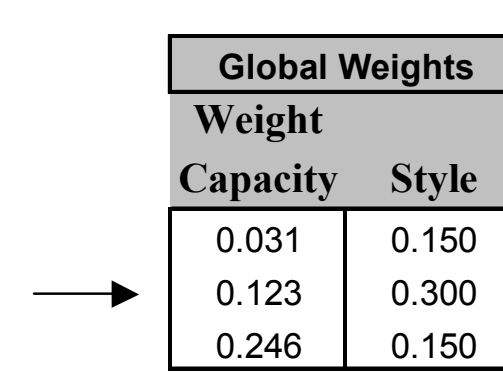

Overall

Priorties

0.181

0.423

0.396

Proceedings $-6^{\text {th }}$ ISAHP 2001 Berne, Switzerland 
converted into global priorities measured in units of the summed criteria weights. In other words, the unit of measure for the global priorities is the total importance of the criterion within the overall hierarchy. Since the sum of the criterion weights equals the overall value of unity for the complete hierarchy, we can also say that the global weights are in the unit of the topmost goal of the hierarchy

The global priorities, measured in the unit of the overall goal, are commensurable within the hierarchy. They can be added to get the composite overall priorities. As can be seen from Table 3, suitcase B with an overall priority of .423 is the most desirable. It was calculated by adding the commensurate global priorities, .123 and .300 . We were unable to simply add the local priorities, .308 and .500 , because they are measured in different units. We were able to add global weights only because they had been converted into a common unit for the whole hierarchy.

In hierarchical form, the answer to our composite problem is given in Figure 1. At the topmost Goal level, the global weight $(\mathrm{GW})$ is equal to unity, indicating that this value is the unit of the entire hierarchy. At the criterion level, only global weights $(\mathrm{GW})$ are shown, because at that level local and global weights are equivalent. Those global criterion weights sum to the unit value of the topmost Goal.

At the alternative level, the local weights (LW) under each triangular area are in the unit of the total criterion possessed by the alternatives of that area. Just like Case 6 of Table 1, the unit for each triangular sub-hierarchy is the sum of the total capacity or style possessed by the alternatives under that criterion. Equivalently, these local priorities (LW) under each criterion sum to one unit of themselves (ie the sum of local priorities possessed by the alternatives under that criterion).

Multiplying the LW by the criteria GW produces the GW of alternatives. They are now measured in the unit of the total hierarchy (as opposed to the unit of a specific criterion). For example, the sum of all global weights at the bottom level equal the unit value of the goal at the top level. For any one alternative, the commensurate GW can be summed to yield that alternative's composite priority that is still in the ratio scale of the overall hierarchy.

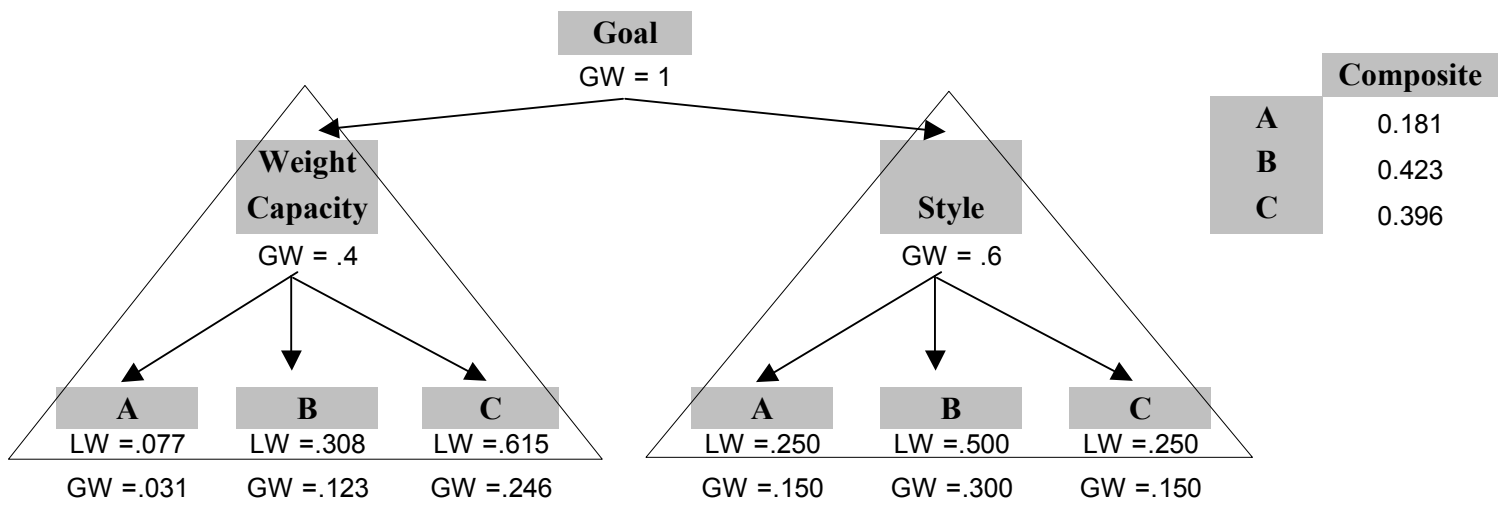

Figure 1 -- AHP Composite Answer in Hierarchical Form

\subsection{Linking Pin Ratios}

In conventional AHP, priorities are normalized to sum to unity, either within a subset of the hierarchy for local weights or within the total hierarchy for global weights of any one level. Ultimately, the composite weights are in the units of the totality of the goal possessed by the attribute measures in the hierarchy. That is by convention. The disadvantage of that convention is that it is not easy to distinguish the common unit in which items are measured. 
Since the unit of measure in ratio scales is arbitrary, there is no need to keep to the unit sum convention. One method that adopts that approach is the linking pin procedure (Schoner et al, 1993), where attribute measure of any alternative, even an aspiration one, can be used as the unit of measure. Figure 2 shows the linking pin results for our sample problem when the weight capacity of suitcase B is used as the link for the entire hierarchy (ie all three levels) and the style of suitcase $\mathrm{C}$ is used as the link for the style criterion. We will explain Figure 2 by working from the top down, to illustrate that linking pin procedures can work top down or bottom up.

At the criterion level, suitcase B for weight capacity and suitcase C for style are used as our comparison referents. They are our links. Since we have chosen the weight capacity of suitcase B as our unit for the overall hierarchy, it will take a value of unity at the criterion level. The criterion weight for style given suitcase $\mathrm{C}$ as the link will take a proportionally larger or smaller amount of the unit. In our case, the style of suitcase $\mathrm{C}$ is estimated to be 1.22 times as important as the weight capacity of suitcase $\mathrm{B}$. We calculated this ratio from Figure 1 - suitcase $\mathrm{C}$ is worth $25 \%$ of a style criterion of $60 \%$ importance (.15) while suitcase B is worth $30.8 \%$ of a capacity criterion worth $40 \%$ importance $(.123)$. The ratio $(.15 / .123)$ is 1.22 .

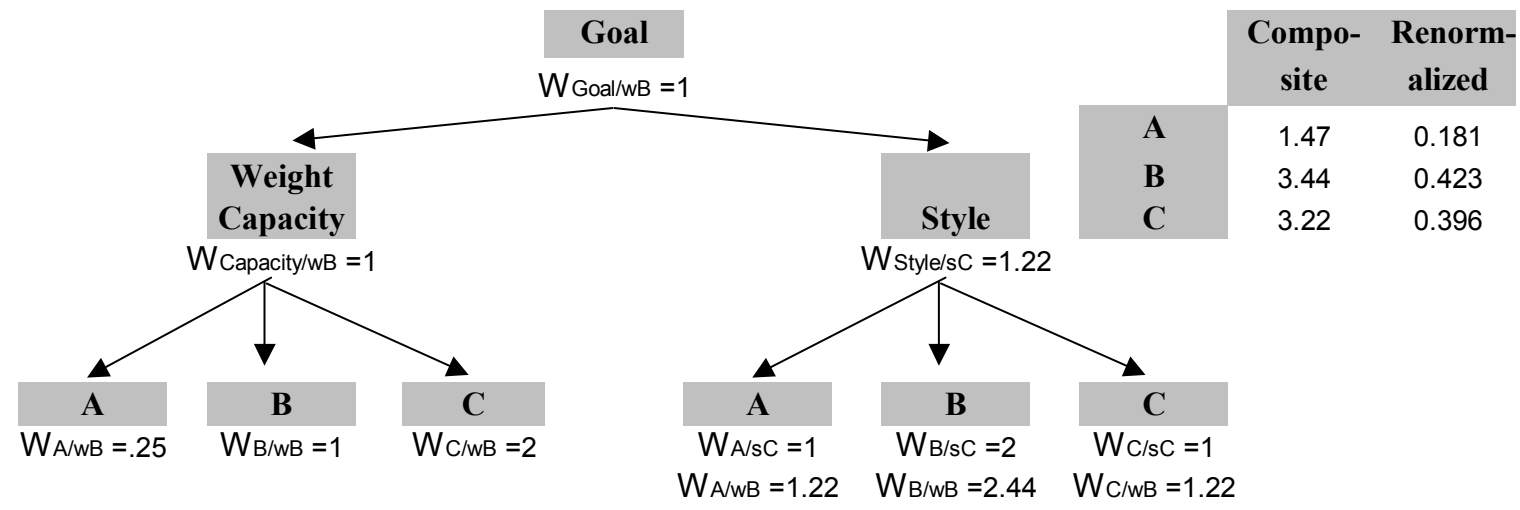

Figure 2 -- Linking Pin Answer in Hierarchical Form

At the alternative level, the attribute measures of linking objects are normalized so that they take a value of unity while the attribute measures of non-linking objects assume proportionally higher or lower values. Since the criterion weights are in units of the weight capacity of suitcase B, hierarchical multiplication transfers that unit value to the lower levels. In the case of the weight capacity criterion, no such multiplication is shown, since the weights are already expressed in terms of the chosen hierarchical unit (weight capacity of suitcase B). As can be seen from Figure 2, the style of suitcase C is worth 1.22 units of the weight capacity of suitcase B. This is the value we determined at the criterion level. The style of suitcase $\mathrm{B}$, being twice as good as suitcase $\mathrm{C}$ takes a worth of 2.44 units of the selected unit of measure.

The bottom ratios in Figure 2 are all in common units (weight capacity of suitcase B). Since they are in the same unit, they can be added together to determine how many units each alternative possesses. As shown, suitcases $\mathrm{A}$. $\mathrm{B}$, and $\mathrm{C}$ have 1.47 units, 3.44 units and 3.22 units respectively. We have re-normalized the results to show that they give the same result as unit-sum normalization.

\subsection{Discussion}

Herein, we have discovered two important points: (1) ratio scales, such as those produced by AHP, have a unit of measure (2) one does not necessarily have to know natural zero and the unit in order to derive a ratio scale. We agree with Barzilai (1998) that all ratio scales have a unit of measure and that the choice of unit is arbitrary. We do not go as far as Barzilai, however, in claiming that natural zero and the unit must be known in order to determine a ratio scale. Although on the surface it may appear contradictory, we agree with Saaty (1999) that derivation of a relative ratio scale does not need a unit of measure. We would not 
claim, however, that a relative ratio scale has no unit. Rather, we claim that knowledge of the unit is important and useful. This discussion is devoted to illumination of these concepts.

In AHP, the process of normalizing to unity obscures the unit of measure. When we get a set of composite results such as those in Table 3, what do we mean when we say that the priorities are in ratio scale? In other words, what is the unit for the composite priorities: .181, .423 and .396 . One way to understand this is to re-express them as a ratio of their unit $(.181 / 1, .423 / 1$ and .396/1) and then ask ourselves the definition of the unit value. When done in this manner, it becomes clearer that the unit (one in the denominator) is the value of the topmost node of the hierarchy.

The derivation of local priorities can be envisaged as a process that determines the unit for a subset of the hierarchy. That unit is the totality (sum) of the specific criterion possessed by the alternatives. For conventional AHP, however, we do not want the unit of a subset of the hierarchy. We want a unit that measures the total hierarchy. To do that, we need to rescale the local priorities by the global importance of the criteria. When that is done, the resulting global priorities are in the unit of the hierarchy.

The obscurity of the unit of measure in conventional AHP leads to many problems. For example, the unit represents the totality of the preference possessed by the attribute measures (criteria and alternatives) included in the hierarchy. If an alternative is added or deleted, then the totality changes and so does the unit. This point is important, because it is often overlooked. It is also the cause of the AHP nemesis -- the rank reversal problem. The addition or deletion of an alternative changes the totality, changes the unit, and changes ratios of the existing attribute measures. If substantial enough, ratio change causes rank change.

People think that AHP's problem is rank instability. As shown herein, the real problem is ratio instability. The dual fact that the unit of measurement is obscure and rank rather than ratio is thought to be important has resulted in the wrong avenue of debate. AHP is ratio measurement and its efficacy should be argued on ratio grounds.

Seeing the ratio relationship is enhanced if the unit of measure is something more tangible. Linking pins provides such a tangible approach. In the linking pin procedure, the attribute measure of a specific alternative is chosen as the unit of measure. Selecting a specific alternative means that the choice of unit no longer becomes arbitrary. The choice of unit thereby becomes clear and evident to all concerned.

Although we agree with Barzilai that ratio measurement has an arbitrary unit of measure, we do not contend that natural zero and the unit must be known in order to make paired comparisons. The only requirement is that the compared items be relevant. By relevant, we mean that the items can be meaningfully perceived or sensed by the decision-maker and that the sensations be within one order of magnitude. In order to perceive attribute measures, it is not necessary for us to know one of the potential units of measure. For example, a person may be given no information about the unit of the three objects measured in Table 1. Without a scale, a person would still be able to say which object is heavier and make judgments about how many times as heavy. Assuming comprehension of multiplication and accuracy in comparisons, we could derive the AHP priorities in the last column of Table 1 without ever knowing one of the underlying units of measure.

And therein lies both the advantage and dilemma of AHP. We do not have to know the underlying unit of measure to derive a ratio scale, yet the derived scale has a unit. Not needing to know the unit, we can derive ratio measurement for attributes that were heretofore unmeasurable (e. g. style, quality, safety, comfort, etc.) Not knowing the unit, we become confused over why rank reversals occur when they are unintended.

Saaty is correct to say that a relative ratio scale does not need a fixed unit of measurement. That, however, is a statement that is relevant for the derivation of the scale. It does not mean that the derived scale is devoid of a unit of measure. The failure to appreciate this fact has caused some users to claim that AHP scales are dimensionless. We do not agree. The scale does have a unit of measure. Being the sum of all attribute measures of the hierarchy, rather than a specific measure as in linking pins, makes the unit 
obscure. Being obscure, it has caused confusion such as the rank reversal debate and the failure of the ideal and distributive mode to produce the same answer for the same problem.

\subsection{Conclusion}

We do not need to know the unit of measure to derive a relative ratio scale, but we need to know it if we are to avoid mistakes. What should we do!

The solution is simple. We must become aware that all ratio scales have a unit of measure, although that unit is arbitrary. Being arbitrary, we can easily change from one unit of measure to another. Proportional transformations such as multiplying by criteria weights achieve that end.

Whatever unit is derived (conventional AHP) or used (Linking Pins), it is important to realize that actions such as adding or deleting alternatives or different normalizations result in a new unit of measure. If additive synthesis is used after such a transformation, it is natural to expect different results if no allowance is made for the change in unit (Choo et al, 1999). It is important, therefore, to realize that AHP priorities are not dimensionless and that all ratio scales have a unit of measurement.

\section{References}

Barzilai, J (1998) "Measurement Foundations for Preference Function Modeling ," Proceedings of the IEEE International Conference on Systems, Man and Cybernetics, 4038-4044.

Choo, E. U., Schoner, B. and Wedley, W. C. (1999) "Interpretation of Criteria Weights in Multicriteria Decision Making, "Computers and Industrial Engineering Journal", 37, 527-541.

Saaty, Thomas L., The Seven Pillars of the Analytic Hierarchy Process, Proceedings of the Fifth International Symposium on the Analytic Hierarchy Process, Kobe, Japan, August 12-14, 1999, 20-33

Saaty, T. L. (1977) “A Scaling Method for Priorities in Hierarchical Structures," Journal of Mathematical Psychology, 15, 3, 234-81.

Saaty, T. L. and Vargas, L.G. (1991) The Logic of Priorities, Pittsburgh: RWS Publications, 1993

Schoner, B., Wedley, W. C. and Choo E. U. (1993) "A unified approach to AHP with linking pins" European Journal of Operations Research, 64,384-392. 
